\title{
Possible involvement of lysosomal dysfunction in pathological changes of the brain in aged progranulin-deficient mice
}

\author{
Yoshinori Tanaka ${ }^{1,2}$, James K Chambers ${ }^{3}$, Takashi Matsuwaki ${ }^{1}$, Keitaro Yamanouchi ${ }^{1}$ and Masugi Nishihara ${ }^{1 *}$
}

\begin{abstract}
Introduction: It has been shown that progranulin (PGRN) deficiency causes age-related neurodegenerative diseases such as frontotemporal lobar degeneration (FTLD) and neuronal ceroid lipofuscinosis (NCL), a lysosomal storage disease. Previous studies also suggested that PGRN is involved in modulating lysosomal function. To elucidate the pathophysiological role of PGRN in the aged brain, in the present study, lysosomal function and pathological changes of the brain were investigated using 10- and 90-week-old wild-type and PGRN-deficient mice.

Results: We showed that PGRN deficiency caused enhanced CD68 expression in activated microglia and astrogliosis in the cortex and thalamus, especially in the ventral posteromedial nucleus/ventral posterolateral nucleus (VPM/VPL), in the aged brain. Immunoreactivity for Lamp1 (lysosome marker) in the VPMNPL and expression of lysosome-related genes, i.e. cathepsin D, V-type proton ATPase subunit d2, and transcription factor EB genes, were also increased by PGRN deficiency. Aggregates of p62, which is selectively degraded by the autophagy-lysosomal system, were observed in neuronal and glial cells in the VPMNPL of aged PGRN-deficient mice. TAR DNA binding protein 43 (TDP-43) aggregates in the cytoplasm of neurons were also observed in aged PGRN-deficient mice. PGRN deficiency caused enhanced expression of glial cell-derived cytotoxic factors such as macrophage expressed gene 1, cytochrome b-245 light chain, cytochrome b-245 heavy chain, complement C4, tumor necrosis factor-a and lipocalin 2. In addition, neuronal loss and lipofuscinosis in the VPMNPL and disrupted myelination in the cerebral cortex were observed in aged PGRN-deficient mice.

Conclusions: The present study shows that aged PGRN-deficient mice present with NCL-like pathology as well as TDP-43 aggregates in the VPMNPL, where a particular vulnerability has been reported in NCL model mice. The present results also suggest that these pathological changes in the VPMNPL are likely a result of lysosomal dysfunction. How PGRN prevents lysosomal dysfunction with aging remains to be elucidated.
\end{abstract}

Keywords: Progranulin, Lysosome, Aging, TAR DNA binding protein 43, Neuronal ceroid lipofuscinosis

\section{Introduction}

Progranulin (PGRN), which is a $68.5-\mathrm{kDa}$ glycoprotein containing 7.5 tandem repeats of a cysteine-rich motif, is proteolytically processed into 6-kDa granulins (GRNs) [1]. PGRN is involved in multiple physiological functions and various disease processes, including autoimmune disorders, tumorigenesis, and insulin resistance [2-4]. In the human brain, haploinsufficiency of PGRN is one of the major factors causing frontotemporal lobar degeneration

\footnotetext{
*Correspondence: amnishi@mail.ecc.u-tokyo.ac.jp

'Department of Veterinary Physiology, Graduate School of Agricultural and Life Sciences, The University of Tokyo, Bunkyo-ku, Tokyo 113-8657, Japan Full list of author information is available at the end of the article
}

(FTLD) [5,6], which is characterized by ubiquitinated cytoplasmic inclusions containing TAR DNA binding protein 43 (TDP-43) [7]. However, the mechanism by which impaired production of PGRN causes the formation of TDP-43 inclusions in the cytoplasm is not yet fully understood.

We previously demonstrated that PGRN expression increases after traumatic brain injury (TBI) in mice, and the major source of PGRN is CD68-positive activated microglia [8]. PGRN deficiency induces exacerbated inflammatory responses that are associated with increased lysosomal biogenesis in activated microglia after TBI. Further, the expression of cytotoxic factors is increased 
in relation to increased lysosomal biogenesis, and neuronal damage is exacerbated in PGRN-deficient mice [9]. Because microglial activation characterized by morphologic and phenotypic changes also occurs with aging [10], increased lysosomal biogenesis and cytotoxic factor production may occur with aging in activated microglia in PGRN-deficient mice. Wils et al. suggested that PGRN-deficient mice have a disrupted autophagy-lysosomal pathway with alterations in lysosomal homeostasis [11].

Patients with a homozygous mutation in the GRN gene present with neuronal ceroid lipofuscinosis (NCL), a group of neurodegenerative lysosomal storage disorders [12]. Additionally, Götzl et al. (2014) recently revealed that FTLD patients due to PGRN deficiency have NCLlike pathology [13]. These reports suggest that disorders with aging from PGRN deficiency occur associated with lysosomal dysfunction. The NCLs display a relatively uniform phenotype in the central nervous system that is characterized by a dramatic loss of cortical neurons, pronounced gliosis, and accumulation of lysosomal autofluorescent lipopigments [14]. NCL model animals show a particular vulnerability early during disease progression in the sensory thalamocortical pathways, i.e., projections from the ventral posteromedial nucleus/ventral posterolateral nucleus (VPM/VPL) of the thalamus to the primary somatosensory barrelfield cortex (S1BF) [15]. Localized aggregation of activated microglia and astrocytes followed by neuronal cell loss occur in this area [16-18]. Impaired myelination also occurs as a feature of some NCL models such as mice deficient in palmitoylprotein thioesterase 1 (Ppt1), ceroid-lipofuscinosis, neuronal $5(C \ln 5)$, and ceroid-lipofuscinosis, neuronal $8(C \ln 8)$ genes $[19,20]$. However, characterization of the brain pathology regarding NCL in the VPM/VPL has yet to be performed in PGRN-deficient mice.

In the present study, the effects of PGRN deficiency on age-associated lysosomal biogenesis and function, inflammatory responses, and TDP-43 aggregates in the cytoplasm were investigated. Further, the brain pathology regarding NCL was also characterized in aged PGRNdeficient mice.

\section{Materials and methods}

\section{Animals}

In this study, 10- and 90-week-old female wild-type (WT) and PGRN-deficient (KO) C57BL/6J mice were used. They were produced from breeding heterozygous pairs in our laboratory and were genotyped using previously described protocols [21]. Mice were maintained under controlled light (lights on, 07:00-19:00), temperature $\left(23 \pm 1^{\circ} \mathrm{C}\right)$, and humidity $(55 \pm 10 \%)$, and were given free access to food and water. All animal experiments were performed in accordance with the Guide for the Care and Use of Laboratory Animals of the University of Tokyo and were approved by the Institutional Animal Care and Use Committee of the University of Tokyo (Permit Number: P12-651).

\section{Antibodies}

The primary antibodies from the following sources were used for immunohistochemistry: mouse monoclonal anti-ubiquitin antibody (MAB1510), rabbit polyclonal anti-microtuble-associated protein 2 (MAP2) antibody (AB5622), and rat monoclonal anti-CD11b antibody (MAB1387Z) from Millipore (Billerica, MA, USA); rabbit polyclonal anti-glial fibrillary acidic protein (GFAP) antibody (Z0334) and rabbit polyclonal anti-myelin basic protein (MBP) antibody (A0623) from Dako (Glostrup, Denmark); rabbit polyclonal anti-TDP-43 antibody (107822-AP) from Proteintech (Chicago, IL, USA); rabbit polyclonal anti-phospho-TDP-43 (Ser409/410) antibody (TIP-PTD-P02) from CosmoBio (Tokyo, Japan); rabbit polyclonal anti-ionized calcium-binding adapter-1 (Iba1) antibody (019-19741) from Wako chemicals (Osaka, Japan); rat monoclonal anti-CD68 antibody (MCA1957GA) from AbD serotec (Oxford, UK); mouse monoclonal anti-MAP2 antibody (M1406) from Sigma (St. Louis, MO, USA); guinea pig polyclonal anti-p62 antibody (03-GP62-C) from American research products (Waltham, MA, USA); rat monoclonal anti-lysosome associated membrane protein 1 (Lamp1) antibody (553792) from BD pharmingen (Franklin Lakes, NJ, USA). Subsequently, the secondary antibodies from following sources were used: biotin-conjugated goat anti-rabbit IgG antibody and biotin-conjugated goat anti-guinea pig IgG from Vector Laboratories (Burlingame, CA, USA); biotin-conjugated donkey anti-rat IgG from Jackson ImmunoResearch Laboratories (West Grove, PA, USA); Alexa Fluor 488 goat anti-mouse IgG antibody, Alexa Fluor 594 goat anti-mouse IgG antibody, Alexa Fluor 594 goat anti-rabbit IgG antibody, Alexa Fluor 594 goat anti-rat IgG antibody, and Alexa Fluor 488 goat anti-guinea pig IgG antibody from Invitrogen (Carlsbad, CA, USA).

\section{Traumatic brain injury (TBI)}

TBI was placed in the cerebral cortex of 10- and 90week-old WT and $\mathrm{KO}$ mice as described previously [8]. Briefly, mice were anesthetized by intraperitoneal administration of xylazine hydrochloride $(8 \mathrm{mg} / \mathrm{kg})$ and chloral hydrate $(300 \mathrm{mg} / \mathrm{kg})$ dissolved in sterile saline and subsequently placed in a stereotaxic apparatus (Narishige, Tokyo, Japan). The right cerebral cortex (1.3 mm lateral to the midline, 1 to $3 \mathrm{~mm}$ posterior to the bregma, $2 \mathrm{~mm}$ in depth) was exposed and injured with a stainless steel cannula with a $0.5-\mathrm{mm}$ outer diameter. Brain samples were taken 4 days after TBI under xylazine hydrochloride and 
chloral hydrate anesthesia or by decapitation, and all efforts were made to minimize suffering.

\section{Tissue preparation for histological analyses}

For immunohistochemical studies, 10- and 90-week-old WT and KO mice ( $\mathrm{n}=5$ for each group) were used. Mice were transcardially perfused with saline followed by $4 \%$ paraformaldehyde in PBS ( $\mathrm{pH}$ 7.2) under xylazine hydrochloride and chloral hydrate anesthesia. Brains were removed from the skull, postfixed in $4 \%$ paraformaldehyde overnight, immersed in 10\% sucrose in PBS for 24 h, $20 \%$ sucrose for $24 \mathrm{~h}$, and $30 \%$ sucrose for $48 \mathrm{~h}$ for cryoprotection, and cut into $30-\mu \mathrm{m}$ sections on a cryostat. Sections were preserved in PBS with $0.01 \%$ sodium azide. Brain sections from bregma -1.46 to -1.82 were used for the analysis. In the present study, we focused on agerelated phenotypical changes in the brain due to PGRN deficiency, and analyzed the uninjured side of the brain unless otherwise mentioned.

\section{Immunochromogenic staining}

Immunochromogenic staining for Iba1, CD68, Lamp1, GFAP, p62, phospho-TDP-43 (pTDP-43) and MBP was carried out on free-floating sections. In brief, sections were incubated in $0.3 \%$ hydrogen peroxide for $30 \mathrm{~min}$, blocked in blocking solution (Block Ace, Snow Brand Milk Products, Sapporo, Japan) at room temperature (RT) for $2 \mathrm{~h}$, and exposed to primary antibody (1:500) at $4^{\circ} \mathrm{C}$ for $60 \mathrm{~h}$. Sections were incubated with biotinylated secondary antibody (1:500) at RT for $2 \mathrm{~h}$, amplified with avidin-biotin complex (Vector Laboratories), and visualized with 3-3'-diaminobenzidine (Sigma) as the chromogen for $1.5 \mathrm{~min}$. All washes were done in $0.03 \%$ PBST, incubations with primary and secondary antibodies were done in $0.3 \%$ PBST containing 1\% BSA, incubations with the avidinbiotin complex were done in 0.3\% PBST. Lamp1-, p62-, and pTDP-43-stained sections were counterstained with cresyl violet solution $(0.2 \%$ cresyl violet, $0.1 \%$ acetic acid in distilled water (DW)). Sections were mounted, dehydrated, and coverslipped with Multi Mount 220 (Matsunami Glass, Osaka, Japan). The sections were then observed under a BX-53 microscope equipped with a DP-73 digital microscope camera (Olympus, Tokyo, Japan).

To measure the immunoreactive (IR) areas, the number of pixels per image with an intensity above the predetermined threshold level was quantified, and the total was calculated using NIH ImageJ software (National Institutes of Health, Bethesda, MD, USA) as the percentage area density, which is defined as the number of pixels divided by the total number of pixels in the imaged field. To determine the number of Iba1-IR cells, cells with a clearly outlined, stained soma were counted. Images on the medial and lateral region along the wound site (injured side) and the comparable contralateral side (uninjured side) were used for the analysis of the cerebral cortex. On the other hand, four images of the left VPM/VPL without overlapping were captured and used for the analysis of VPM/ VPL, and the values of the four images were averaged. For analysis of the number of Iba1-IR cells in layers IV of the S1BF, an image on the left S1BF was used. A rectangular box $(400 \times 150 \mu \mathrm{m})$ was localized to layers IV of the S1BF, and the number of Iba1-IR cells was counted. To measure fractal dimension (FD) of Iba1-IR cells, ten images of Iba1-IR cells of the left cerebral cortex and VPM/VPL were used for analysis. FD was calculated by the software (PopImaging v5.00, the trial version, http://www.dbkids. co.jp), and the value of the ten images was averaged.

\section{Immunofluorescent staining}

Immunofluorescent staining for MAP2, GFAP, MBP, CD68, p62, ubiquitin, and TDP-43 was carried out on free-floating sections. To improve specific fluorescent signals and reduce autofluorescence, antigen retrieval and Sudan black B treatment was performed as described previously [22]. Briefly, sections were incubated in $10 \mathrm{mM}$ citrate buffer ( $\mathrm{pH}$ 6.0) at $90^{\circ} \mathrm{C}$ for $30 \mathrm{~min}$, followed by incubation in $0.1 \%$ Sudan black B (Wako Pure Chemicals) in 70\% ethanol for $20 \mathrm{~min}$ at RT. The sections were blocked in blocking solution (Block Ace, Snow Brand Milk Products) at RT for $2 \mathrm{~h}$ and exposed to primary antibody at $4^{\circ} \mathrm{C}$ for $60 \mathrm{~h}$. Sections were incubated with Alexa Fluor 488- or 594conjugated secondary antibody (1:500) at RT for $2 \mathrm{~h}$. Nuclei were stained with Hoechst33258 $(0.1 \mu \mathrm{g} / \mathrm{ml}$; Polysciences Inc., Warrington, PA) at RT for $30 \mathrm{~min}$. All sections were mounted on glass slides and coverslipped with fluoromount (Diagnostic BioSystems, Pleasanton, CA). To analyze the localization of p62, the sections were examined under an LSM 510 laser scanning confocal microscope (Carl Zeiss, Oberkochen, Germany). For triple staining for p62, ubiquitin, and Hoechst33258; p62, TDP43, and Hoechst33258, the sections were examined under an LSM 5 PASCAL laser scanning confocal microscope (Carl Zeiss).

\section{Real-time PCR}

The left thalamus was dissociated from 90-week-old WT $(\mathrm{n}=6)$ and KO mice $(\mathrm{n}=5)$ using tweezers with a fine tip. Total RNA was isolated from the samples with TRIzol (Invitrogen), and first-strand cDNA was synthesized with SuperScriptII reverse transcriptase (Invitrogen). PCR reactions for cathepsin D (Ctsd), V-type proton ATPase subunit d 2 (Atp6v0d2), transcription factor EB (Tfeb), macrophage expressed gene 1 (Mpeg1), cytochrome b-245 light chain $(C y b a)$, cytochrome b-245 heavy chain $(C y b b)$, complement $\mathrm{C} 4(C 4)$, tumor necrosis factor $\alpha$ $(T N F-\alpha)$, lipocalin 2 (Lcn2), p62, and hypoxanthine phosphoribosyltransferase (Hprt; an internal standard) were performed with Thunderbird SYBR qPCR MIX (Toyobo, 
Osaka, Japan), a LightCycler (Roche, Mannheim, Germany), and each primer set listed in Additional file 1: Table S1. The PCR reactions for Hprt were carried out as follows: $1 \mathrm{~min}$ at $95^{\circ} \mathrm{C}$ for the initial denaturation, followed by 40 cycles of amplification at $95^{\circ} \mathrm{C}$ for $15 \mathrm{~s}, 67^{\circ} \mathrm{C}$ for $20 \mathrm{~s}$, and $72^{\circ} \mathrm{C}$ for $15 \mathrm{~s}$. For other primers, the PCR conditions were: $1 \mathrm{~min}$ at $95^{\circ} \mathrm{C}$ for the initial denaturation, followed by 40 cycles of amplification at $95^{\circ} \mathrm{C}$ for $15 \mathrm{~s}$ and $60^{\circ} \mathrm{C}$ for $60 \mathrm{~s}$. The genes of interest were normalized to the expression level of Hprt.

\section{Assessment of viable neurons}

Viable neurons were visualized using Nissl staining. For Nissl staining, the brain sections were mounted on slides and dried at RT. The slides were incubated with Nissl staining solution $(0.2 \%$ cresyl violet, $0.1 \%$ acetic acid in DW) for $30 \mathrm{~min}$. The slides were washed with DW, dehydrated, and coverslipped with Multi Mount 220 (Matsunami Glass). The sections were then observed under a BX-53 microscope equipped with a DP-73 digital microscope camera (Olympus). For analysis of the number of viable neurons in layers IV, V, and VI of the S1BF, an image on the left S1BF was used. A rectangular box $(400 \times 150 \mu \mathrm{m})$ was localized to layers IV, V, and VI of the S1BF, and the number of viable neurons was counted. For analysis in the VPM/ VPL, four images without overlapping were captured, and the number of viable neurons was counted in each image and averaged. A viable neuron was defined as a neuron not exhibiting cytoplasmic shrinkage or nuclear pyknosis.

\section{Lipofuscin analysis}

For autofluorescence analysis, brain sections were mounted on slides, dried at RT, and coverslipped with fluoromount (Diagnostic BioSystems). The sections were then observed under a BX-53 microscope equipped with a DP-73 digital microscope camera (Olympus). The CA3 area was isolated from the hippocampal image using digital image manipulation software (GIMP v2.8.0, Open source software, http://www.gimp.org/) and used for analysis. Four images were captured to avoid overlap with each other on the VPM/VPL. To measure the autofluorescent areas, the number of pixels per image with an intensity above the predetermined threshold level was quantified, and the total was calculated using $\mathrm{NIH}$ Image J software as the percentage area density, which is defined as the number of pixels divided by the total number of pixels in the imaged field. Further, the brown-yellow pigment in the cytoplasm was evaluated with hematoxylin staining, PAS reactivity after diastase treatment, reducing substances by Schmorl staining, and sudanophilia by Oil-red-O or Sudan black B staining.

\section{Assessment of myelination}

Myelination was visualized using avidin-biotin-peroxidase complex method with diaminobenzidine as the chromogen as described in the immunochromogenic staining. The sections were observed under a BX-53 microscope equipped with a DP-73 digital microscope camera (Olympus). To analyze myelination in the temporal association cortex (TeA) and ectorhinal cortex (Ect), an image was captured on the left TeA and Ect, and the image was binarized using digital image manipulation software (GIMP v2.8.0). After that, the distance from the terminal of MBP-IR area to the rim of the cortex in the TeA and Ect was investigated using digital image manipulation software (GIMP v2.8.0).

\section{Statistical analyses}

The data were analyzed with an unpaired $t$-test or twoway ANOVA followed by a Tukey-Kramer test. Data are expressed as the mean \pm SEM. Differences were considered statistically significant when $\mathrm{P}<0.05$.

\section{Results \\ PGRN deficiency leads to enhanced CD68 expression in activated microglia}

We first performed immunohistochemistry for Iba1, a marker for microglia, and compared the morphology and number of Iba1-IR cells between 10- and 90-weekold WT and KO mice (Figure 1). Conspicuous accumulation of Iba1-positive cells was observed in the thalamus, especially in the VPM/VPL, on the uninjured side of 90-week-old KO mice (Figure 1A). We therefore focused on both the thalamus and the cerebral cortex in this study. High-power images revealed that Iba1-IR cells in 10-week-old mice were characterized by a small soma and numerous thin, branched processes (ramified), whereas those in 90-week-old mice had a larger soma with fewer and shorter processes in both WT and $\mathrm{KO}$ mice (Figure 1A), suggesting that microglia were activated with aging. Indeed, FD, which is the index for the complexity of the morphology, of Iba1-IR cells in WT and KO mice was decreased with aging both in the cortex and VPM/VPL, while FD was not different between 90-week-old WT and KO mice both in the cortex and VPM/VPL (Figure $1 \mathrm{~B}$ and $\mathrm{C}$ ). On the other hand, in the cortex, the number of Iba1-IR cells was not different among the groups (Figure 1D). In the VPM/VPL, the number of Iba1-IR cells was not different between 10-week-old WT and KO mice, but significantly larger in $\mathrm{KO}$ mice than WT mice at 90 weeks (Figure 1E).

Immunohistochemical images for CD68, a member of the Lamp family expressed in microglia, in the cerebral cortex and VPM/VPL are shown in Figure 2A. The CD68-IR area was not significantly different between 10week-old WT and KO mice, but significantly larger in 

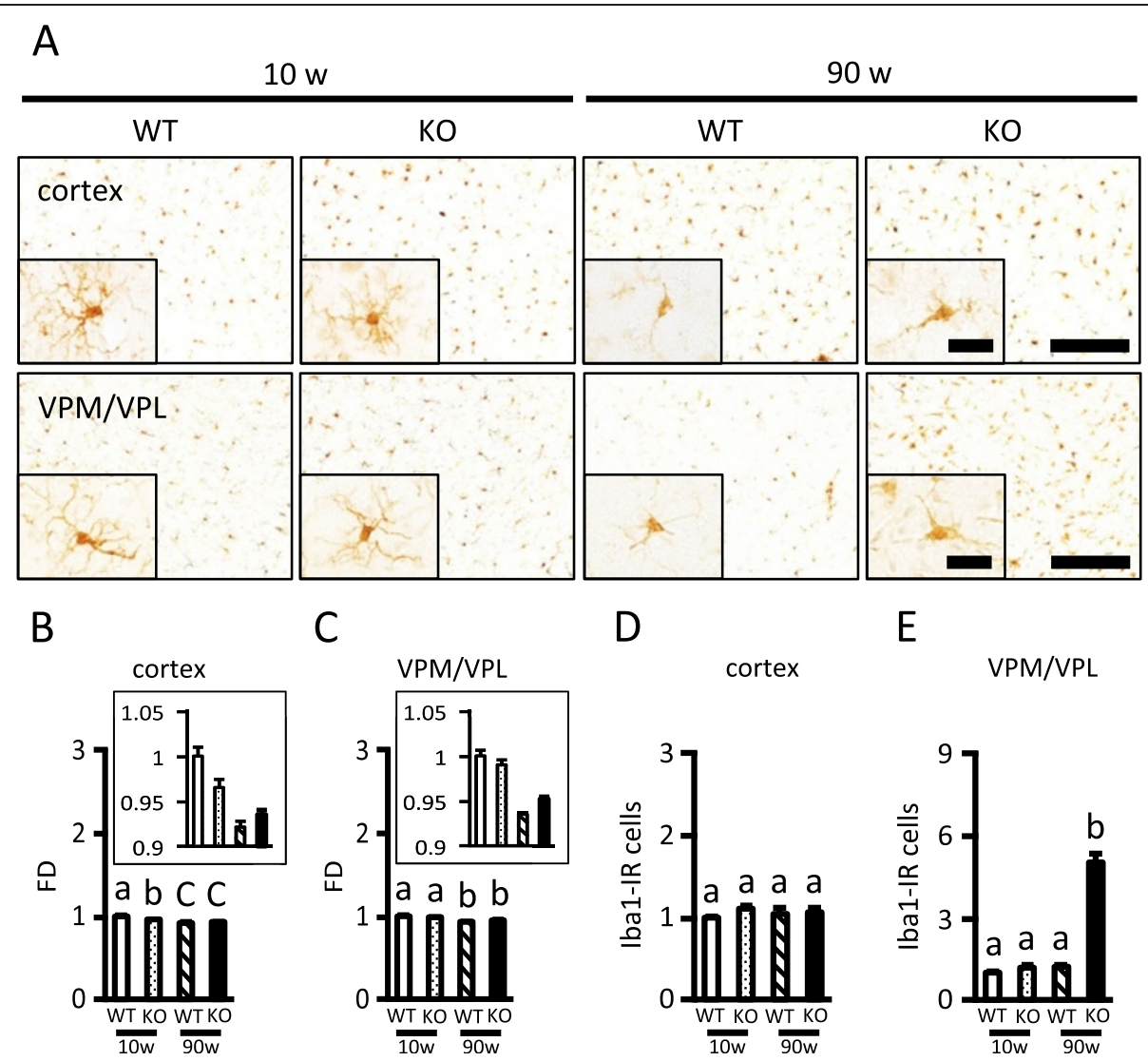

Figure 1 Increased Iba1-IR cells in the VPM/VPL of aged PGRN-deficient mice. $\boldsymbol{A}$, Iba1-immunostained images of the cerebral cortex (upper images) and the VPMNPL (lower images) of 10- and 90-week-old wild-type (WT) and PGRN-deficient (KO) mice. The magnified view is shown on the lower-left corner of each image. Low-power field: scale bar $=200 \mu \mathrm{m}$. High-power field: scale bar $=25 \mu \mathrm{m}$. $\boldsymbol{B}, \boldsymbol{C}$, The fractal dimension (FD) of Ibal-IR cells in the cerebral cortex $(\boldsymbol{B})$ and VPMNPL $(\boldsymbol{C})$ of 10- and 90-week-old WT and KO mice. The figure in a box shows the magnified view of the corresponding figure. The data are presented as the change relative to the number in 10-week-old WT mice. Values are the mean \pm SEM ( $n=5$ for each group). Values having a different superscript are significantly different $(P<0.05$, Tukey-Kramer test). $\boldsymbol{D}, \boldsymbol{E}$, The number of Iba1-IR cells in the cerebral cortex $(\boldsymbol{D})$ and VPMNPL $(\boldsymbol{E})$ of 10- and 90-week-old WT and KO mice. The data are presented as the change relative to the number in 10-week-old WT mice. Values are the mean \pm SEM ( $n=5$ for each group). Values having a different superscript are significantly different $(P<0.05$, Tukey-Kramer test).

90-week-old KO mice than WT mice in both the cortex and VPM/VPL (Figure 2C and D). Additionally, CD68IR cells in the VPM/VPL in PGRN-deficient mice were round or amoeboid cells (Figure $2 \mathrm{~B}$ ). These results suggest that PGRN deficiency enhances CD68 expression in activated microglia in both the cerebral cortex and VPM/VPL of the aged brain, although microgliosis with aging was more prominent in the VPM/VPL.

\section{PGRN deficiency leads to an increase in lysosomal biogenesis}

We then assessed lysosomal biogenesis using Lamp1 (lysosome marker) immunostaining in the layer $\mathrm{V}$ of the cerebral cortex and the VPM/VPL of the thalamus (Figure 3). The Lamp1-IR area was significantly increased with aging in both the layer V and VPM/VPL. Lamp1 immunoreactivity in the layer $\mathrm{V}$ was not different between WT and KO mice at 10 weeks, but was significantly higher in $\mathrm{KO}$ mice than WT mice at 90 weeks of age (Figure 3A, B). Similarly, Lamp1 immunoreactivity in the VPM/VPL was not different between WT and $\mathrm{KO}$ mice at 10 weeks, but was significantly higher in KO mice than WT mice at 90 weeks of age (Figure 3A, C). Further, we compared gene expressions of the lysosomal enzyme $(C t s d)$, protein $(A t p 6 v 0 d 2)$ and the master regulator of lysosomal gene expression $(T f e b)$ in the thalamus between 90-week-old WT and KO mice. These gene expressions in $\mathrm{KO}$ mice were significantly increased compared to expression in WT mice (Figure 3D).

\section{PGRN deficiency leads to an increase in astrogliosis}

Next, we performed immunohistochemistry for GFAP, a marker of astroglia (Figure 4). In both the cerebral cortex and VPM/VPL, the GFAP-IR area was significantly increased with aging. Although the GFAP-IR area was not different between WT and KO mice at 10 weeks, the 


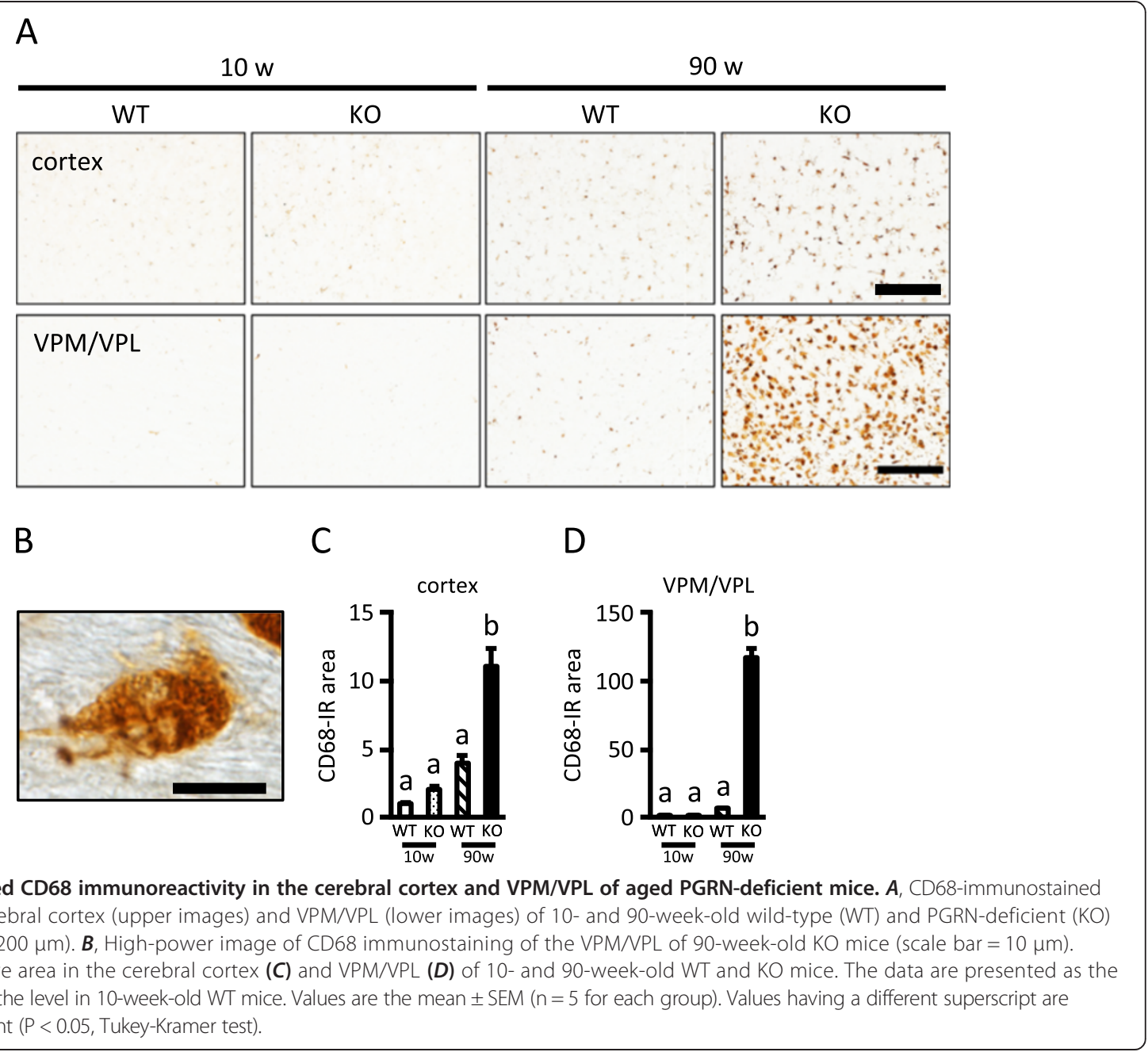

GFAP-IR area was significantly larger in $\mathrm{KO}$ than WT mice at 90 weeks of age in both areas (Figure 4A, C, and D). As shown in Figure 4B, GFAP-IR astrocytes in the VPM/VPL of 90-week-old WT mice showed variable degrees of cellular hypertrophy, suggesting mild to moderate reactive astrogliosis. On the other hand, GFAP-IR astrocytes in 90-week-old KO mice had thicker processes, suggesting severe diffuse reactive astrogliosis [23].

PGRN deficiency results in neuronal loss in the VPM/VPL and increased microgliosis in the S1BF layer IV

We concentrated our analysis on the VPM/VPL and the S1BF. Neurons in the VPM/VPL project to the S1BF, and both of these structures show particular vulnerability in previously reported NCL mouse models [15]. We performed Nissl staining to compare the number of viable neurons in the VPM/VPL in 10- and 90-week-old WT and KO mice. The number of viable neurons was not different among 10-week-old WT, 10-week-old KO, and 90-week-old WT mice, but significantly decreased in 90-week-old $\mathrm{KO}$ mice (Figure 5A and B). Next, we examined gene expression of glial cell-derived cytotoxic factors, i.e., Mpeg1, Cyba, Cybb, C4, TNF- $\alpha$, and Lcn2 in the left thalamus in 90-week-old mice. The expression of all of these genes was significantly higher in $\mathrm{KO}$ mice than in WT mice (Figure 5C). On the other hand, neuronal loss was not detected in 90-week-old $\mathrm{KO}$ mice in layers IV, V, and VI of the S1BF (data not shown). However, the number of Iba1-positive cells in layer IV of the S1BF, which receives projections from the VPM/ VPL, was significantly increased only in 90-week-old $\mathrm{KO}$ mice (Figure 5D and E). These results suggest that aged PGRN-deficient mice show particular vulnerability in the VPM/VPL at least partially due to increased expression of cytotoxic factors by glial cells.

\section{PGRN deficiency results in impaired cellular degradation in lysosomes}

To investigate cellular degradation in lysosomes, we performed immunohistochemistry for p62, which is selectively degraded by the autophagy-lysosomal pathway, in 10 and 90-week-old WT and KO mice. Considerable p62- 
A

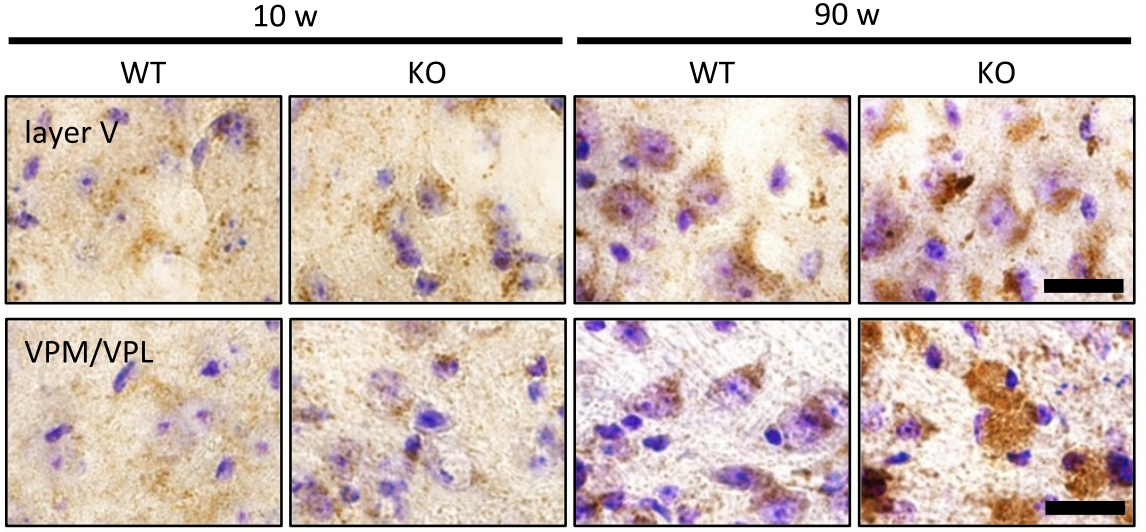

B

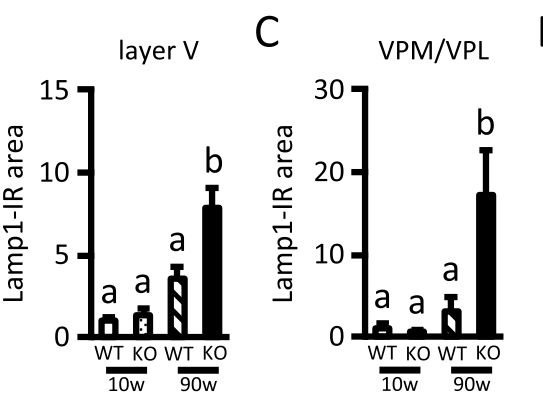

D

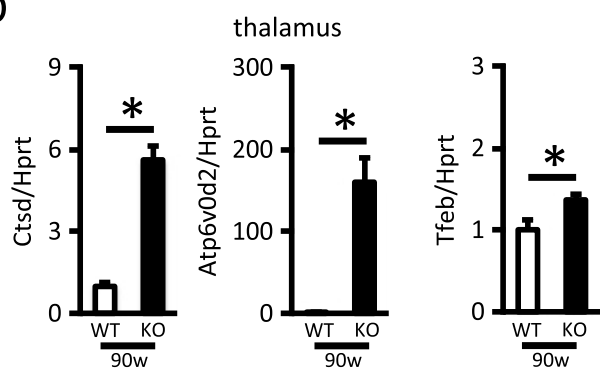

Figure 3 Increased lysosomal biogenesis in the cerebral cortex and VPM/VPL of aged PGRN-deficient mice. A, Lamp1-immunostained images of the layer $V$ of the cerebral cortex (upper images) and VPMNPL (lower images) of 10- and 90-week-old wild-type (WT) and PGRN-deficient $(\mathrm{KO})$ mice (scale bar $=20 \mu \mathrm{m}) . \boldsymbol{B}, \boldsymbol{C}$, Lamp1-positive area in the layer $\mathrm{V}$ of the cerebral cortex (B) and VPMNPL (C) of 10- and 90-week-old WT and KO mice. The data are presented as the change relative to the level in 10-week-old WT mice. Values are the mean \pm SEM $(n=5$ for each group). Values having a different superscript are significantly different $(P<0.05$, Tukey-Kramer test). $\boldsymbol{D}$, Gene expressions of Ctsd, Atp6r0d2, and Tfeb in 90-week-old WT and $\mathrm{KO}$ mice. The data are presented as the change relative to the level in WT mice. Values are the mean $\pm \operatorname{SEM}(n=6$ for $W T ; n=5$ for $K O$, *P $<0.05$, unpaired t-test).

positive aggregates in the VPM/VPL were observed only in 90-week-old KO mice (Figure 6A). The p62-IR area in the VPM/VPL of 90-week-old mice was significantly larger in KO mice than WT mice (Figure 6B), although gene expression for p62 was not different between 90-week-old WT and KO mice (Figure 6C). Because a high-power image suggested that p62-positive inclusions were present in both neuronal and glial cells (Figure 6D), we next investigated what types of cells in the VPM/VPL accumulated p62 using double immunostaining for p62 and MAP2 (neuronal marker), GFAP (astrocyte marker), MBP (oligodendrocyte marker), and CD68 (microglia marker; mainly localized to late endosomes and lysosomes). As shown in Figure 6E, p62 was occasionally colocalized with MAP2, GFAP, MBP, and CD68. In addition, some p62-positive inclusions were also positive for ubiquitin (Figure 6F). These results suggest that PGRN deficiency leads to disruption of the autophagy-lysosomal system in neuronal and glial cells.
PGRN deficiency leads to TDP-43 aggregation in the cytoplasm of neurons and lipofuscin accumulation in microglia

We next performed TDP-43 immunostaining in the VPM/VPL in 10 and 90-week-old WT and KO mice. TDP-43 immunoreactivity was decreased in 90-week-old $\mathrm{KO}$ mice compared with other groups (Figure 7A), probably due to neuronal loss (see Figure 5A). Triple staining for TDP-43, p62, and Hoechst33258 showed that the TDP-43 aggregate colocalized with p62 in the cytoplasm was observed only in aged $\mathrm{KO}$ mice (Figure $7 \mathrm{~B}$ ). Triple staining for TDP-43, MAP2, and Hoechst33258 further showed that the TDP-43 aggregate was present in the cytoplasm of neurons in aged $\mathrm{KO}$ mice (Figure $7 \mathrm{C}$ ). Additionally, pTDP-43-IR aggregate was observed in aged $\mathrm{KO}$ mice (Figure 7D).

To estimate lipofuscin accumulation, autofluorescence in the cerebral cortex, hippocampus, and thalamus was evaluated. Autofluorescence was not detected in 10week-old WT and KO mice (data not shown). On the 


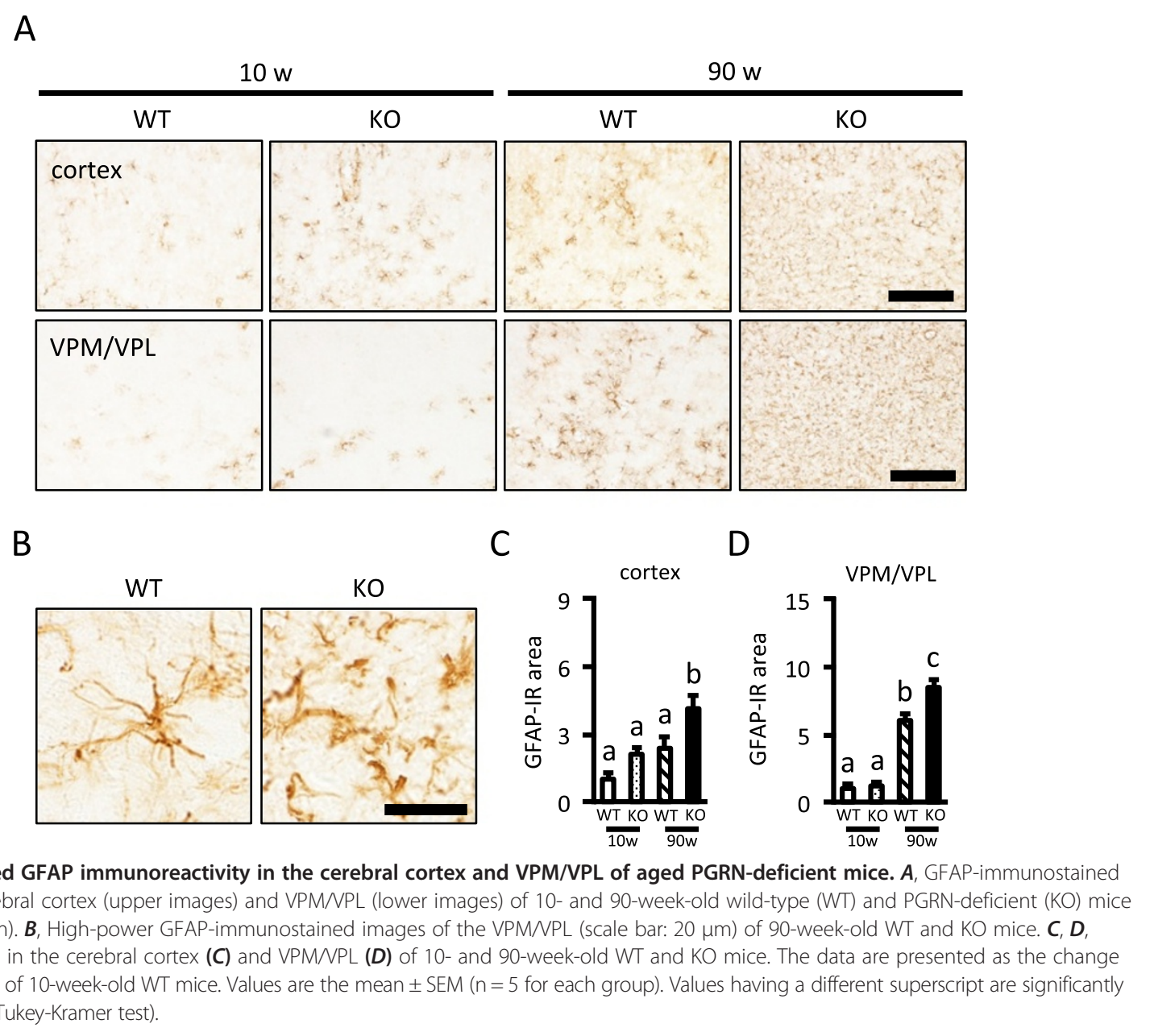

other hand, at 90-week-old, autofluorescence was similar in the cerebral cortex and the CA3 of the hippocampus between WT and KO mice, whereas it was significantly increased in $\mathrm{KO}$ mice compared with WT mice in the VPM/VPL (Figure 8A, B). Lipofuscin represents an intracellular lipopigment of lysosomal origin and appears in unstained sections as brown-yellow autofluorescent intracellular granules that are positive for periodic acidSchiff (PAS), Schmorl, and Oil-red-O staining [24]. A brown-yellow pigment was observed in the cytoplasm (Figure 8C), and reactivity for Schmorl (Figure 8D), PAS (Figure $8 \mathrm{E}$ ), and Oil-red-O (Figure $8 \mathrm{~F}$ ) was positive in the VPM/VPL in 90-week-old KO mice. Additionally, double staining with Sudan black B and CD11b (microglia marker; localized to the plasma membrane) revealed that lipofuscin was accumulated in microglia in the VPM/VPL of KO mice (Figure 8G).

\section{PGRN deficiency results in impaired myelination in the cerebral cortex}

NCL mouse models such as Ppt1-, Cln5-, and $C \ln 8$-deficient mice exhibit a loss of myelin as one of the typical disease features $[19,20]$. We therefore performed immunohistochemical staining for MBP, a major protein constituent of the myelin sheath that surrounds axons and is synthesized by oligodendrocytes [25], using 10 and 90week-old WT and KO mice. Compared to 90 -week-old WT mice, MBP immunoreactivity in cerebral cortex, especially on the lateral side including the TeA and Ect, was relatively disorganized in 90-week-old $\mathrm{KO}$ mice (Figure 9A). The distance from the terminal of MBP-IR area to the rim of the cortex in the TeA and Ect was lessened with aging in WT mice, suggesting myelin was formed with aging. On the other hand, in $\mathrm{KO}$ mice, the distance was not different with aging, suggesting myelin formation was disturbed (Figure 9B).

\section{Discussion}

The present study demonstrated that PGRN deficiency leads to enhanced lysosomal biogenesis in the cerebral cortex and thalamus, especially in the VPM/VPL, with aging. Additionally, we showed impaired lysosomal function and the TDP-43 aggregate in the cytoplasm of neurons in the VPM/VPL in aged PGRN-deficient mice. 
A
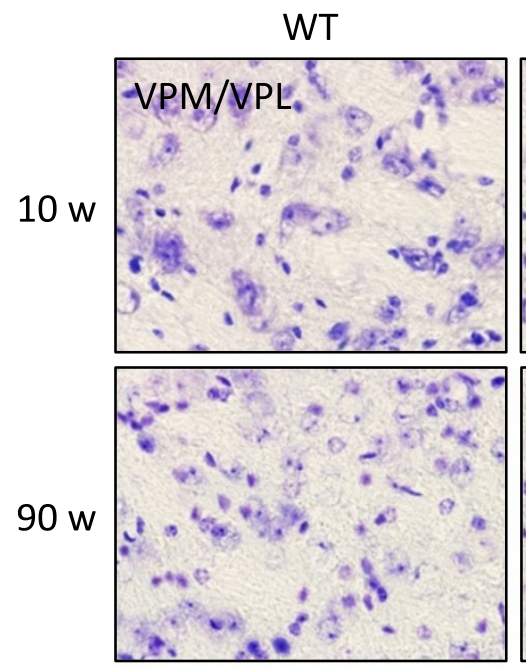
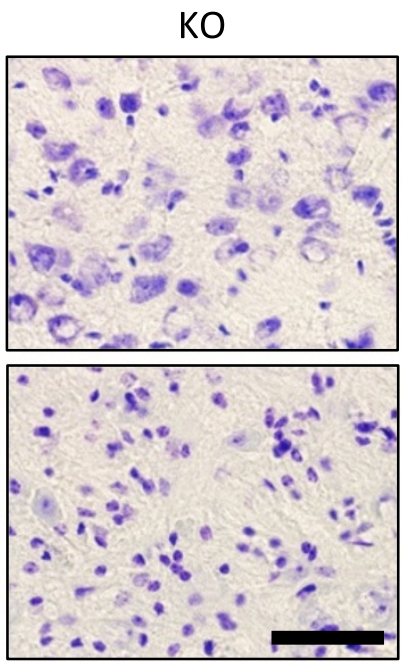

B

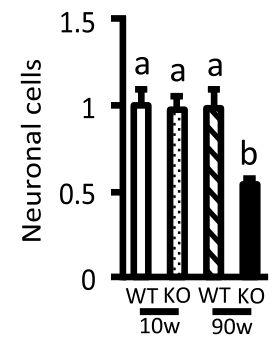

C
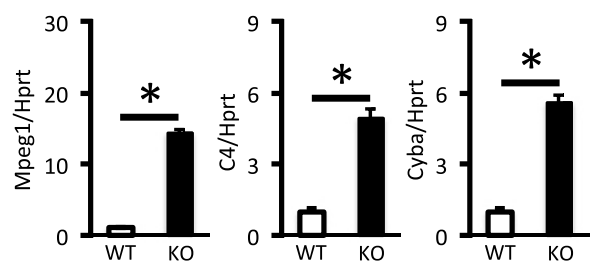

D

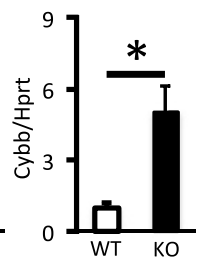

WT KO
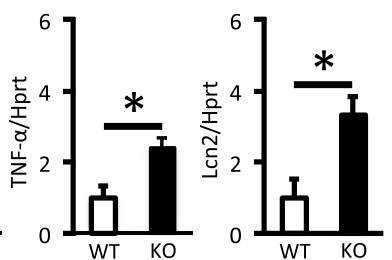

$E$
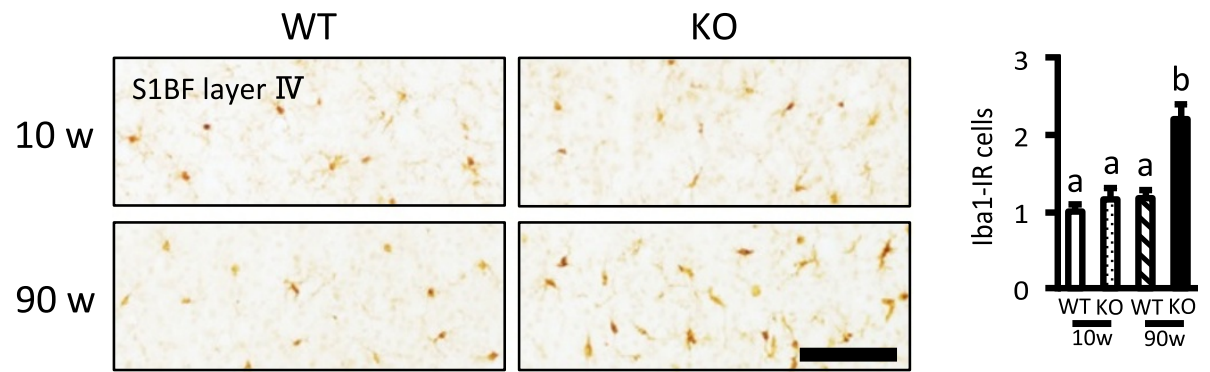

Figure 5 Neuronal loss and increased expression of cytotoxic factors in the VPM/VPL of aged PGRN-deficient mice. $A$, Nissl-stained images of the VPM/VPL of 10- and 90-week-old WT and KO mice (scale bar $=50 \mu \mathrm{m}$ ). $\boldsymbol{B}$, The number of Nissl-positive cells in the VPMNPL of 10- and 90-week-old WT and KO mice. The data are presented as the change relative to the number of 10-week-old WT mice. Values are presented as the mean \pm SEM ( $n=5$ for each group). Values having a different superscript are significantly different $(P<0.05$, Tukey-Kramer test). $C$, Comparison of gene expression for Mpeg1, C4, Cyba, Cybb, TNF- $a$, and Lcn2 between 90-week-old WT and KO mice in the thalamus. The data are presented as the change relative to the level in WT mice. Values are the mean \pm SEM ( $n=6$ for WT; $n=5$ for KO, ${ }^{*} P<0.05$, unpaired t-test). $\boldsymbol{D}$, Iba1-immunostained images in the layer IV of the S1BF of 10- and 90-week-old WT and KO mice (scale bar $=100 \mu \mathrm{m}$ ). $\boldsymbol{E}$, The number of Iba1-IR cells in the VPMNPL of 10- and 90-week-old WT and KO mice. The data are presented as the change relative to the number of 10-week-old WT mice. Values are presented as the mean \pm SEM ( $n=5$ for each group). Values having a different superscript are significantly different $(P<0.05$, Tukey-Kramer test).

Further, aged PGRN-deficient mice developed NCLlike pathology. Thus, although the possibility that contusion in the unilateral cerebral cortex might affect the severity of histological changes and the levels of gene expressions in the contralateral cortex and the thalamus could not be ruled out, substantial pathological changes were observed in the aged brain due to PGRN deficiency.
In our previous study, we showed that although the total number of Iba1-IR microglia was not different between WT and KO mice, CD68 immunoreactivity was significantly higher in young (8- or 9-week-old) KO than WT mice that underwent TBI [8]. In addition, we have shown that PGRN deficiency leads to decreased mammalian target of rapamycin complex 1 (mTORC1) activity with a resultant increase in lysosomal biogenesis in 


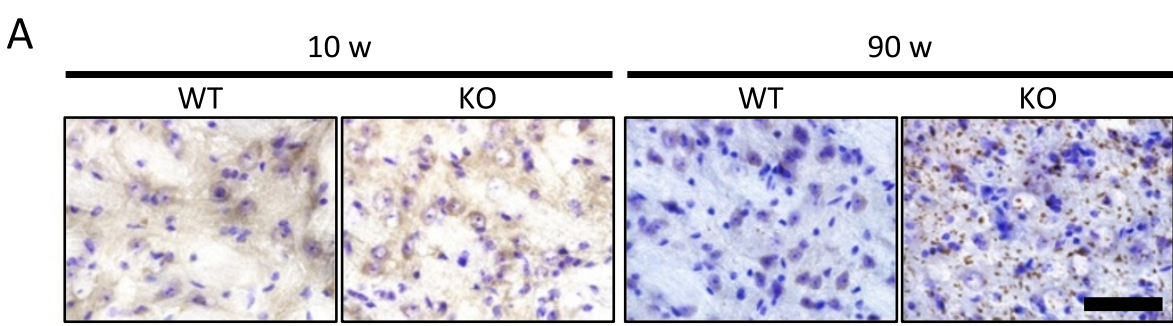

B

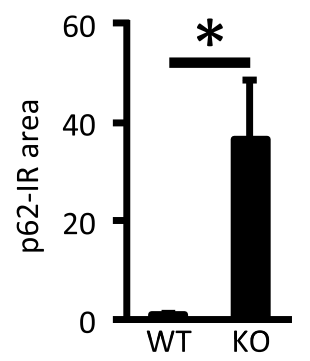

C

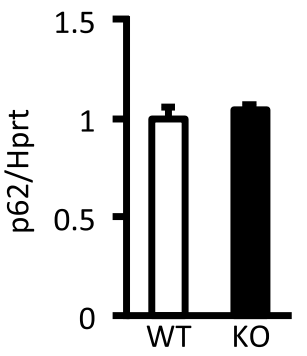

D

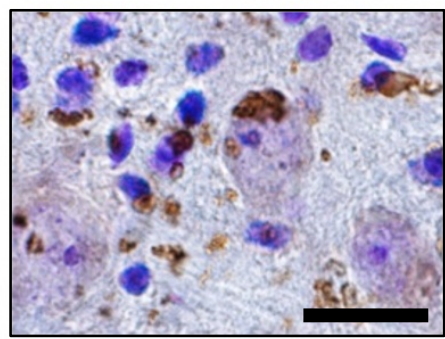

E

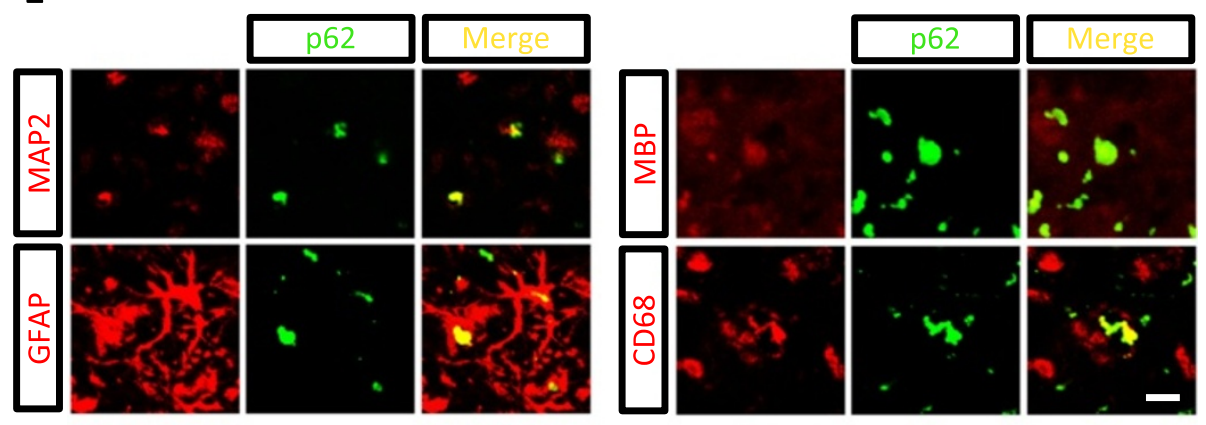

F

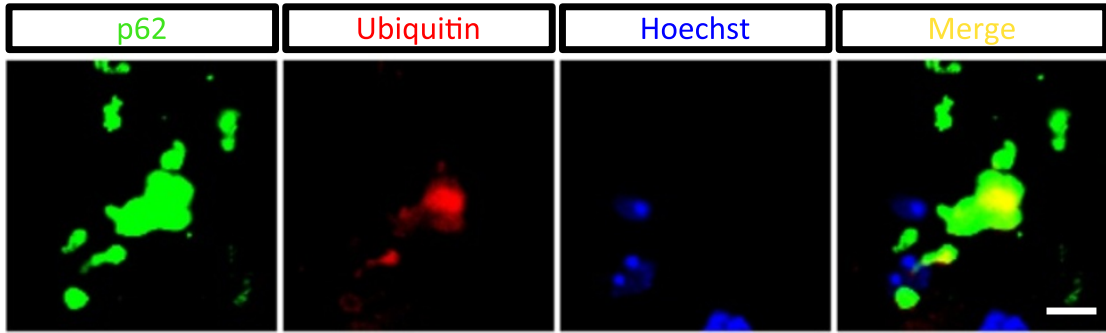

Figure 6 Excessive p62 accumulation in the VPM/VPL of PGRN-deficient mice with aging. $\boldsymbol{A}$, p62-immunostained images with Nissl counterstaining in the VPM/VPL of 10- and 90-week-old WT and KO mice (scale bar $=100 \mu \mathrm{m})$. $\boldsymbol{B}, \boldsymbol{C}$, p62-positive area (B) and p62 gene expression (C) in the VPM/VPL of 90-week-old WT and KO mice. The data are presented as the change relative to the level of WT mice. Values are the mean \pm SEM ( $\boldsymbol{B}: \mathrm{n}=5$ for each group; $\boldsymbol{C}: \mathrm{n}=6$ for $\mathrm{WT}, \mathrm{n}=5$ for $\mathrm{KO}$, ${ }^{*} \mathrm{P}<0.05$, unpaired t-test). $\boldsymbol{D}$, High-power p62-immunostained image with Nissl counterstaining (scale bar $=20 \mu \mathrm{m}$ ). $\boldsymbol{E}$, Double-stained images of p62 (green) with MAP2 (red), GFAP (red), MBP (red), and CD68 (red) in the VPMNPL of 90-week-old KO mice (scale bar $=10 \mu \mathrm{m}$ ). $\boldsymbol{F}$, The triple-stained image of p62 (green), ubiquitin (red) and Hoechst33258 (blue) in the VPMNPL of 90-week-old $\mathrm{KO}$ mice (scale bar $=10 \mu \mathrm{m}$ ).

activated microglia after TBI [9]. Using aged (90-weekold) mice, the present study showed that both the Lamp1 and CD68 immunoreactivity in the cerebral cortex were significantly higher in $\mathrm{KO}$ than in WT mice, even in the absence of TBI, whereas the number of Iba1-IR microglia was not different between the two genotypes. Thus, we made similar observations in the TBI and aging models, both of which are associated with microglial activation and microgliosis. PGRN is therefore likely to be primarily implicated in lysosomal biogenesis that leads to microglial activation and microgliosis. 
A

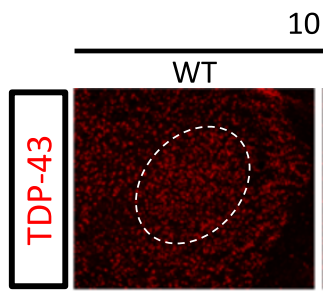

$10 \mathrm{w}$

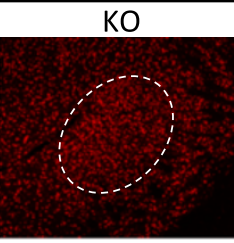

B
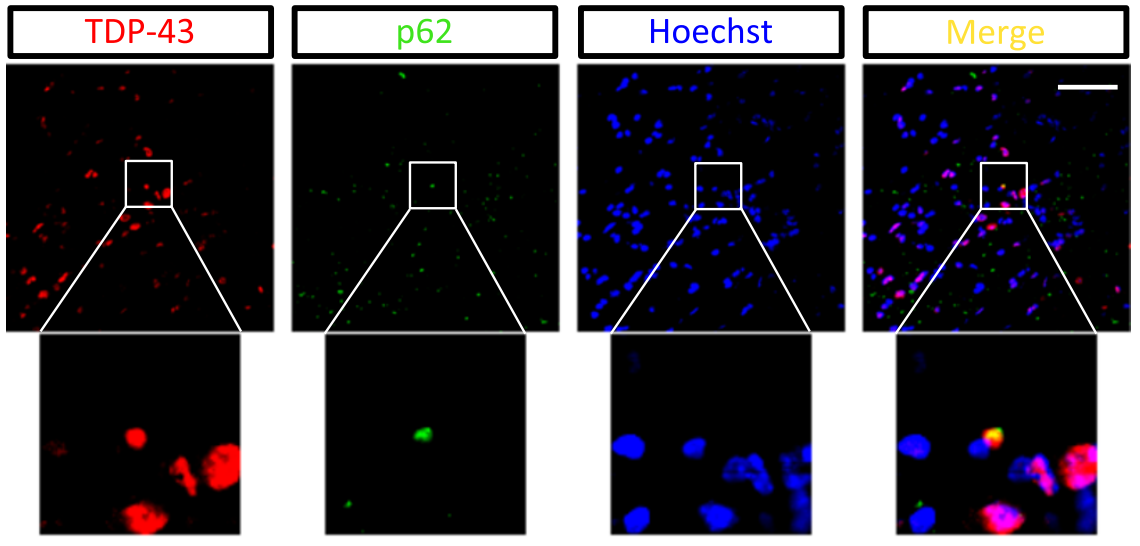

C
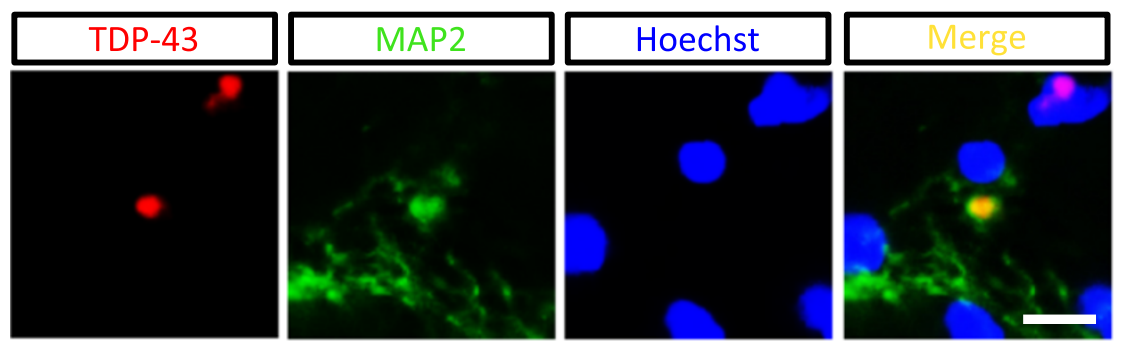

D
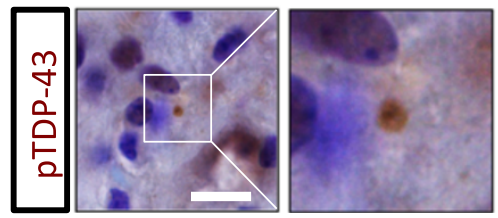

Figure 7 TDP-43 aggregation in the cytoplasm of neurons in the VPM/VPL of aged PGRN-deficient mice. $\boldsymbol{A}$, TDP-43-immunostained images in the VPMNPL of 10- and 90-week-old WT and KO mice (scale bar $=500 \mu \mathrm{m}$ ). The area enclosed by dashed line indicates the VPM/VPL.

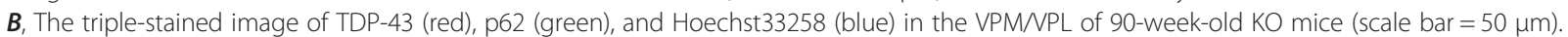
C, The triple-stained image of TDP-43 (red), MAP2 (green), and Hoechst33258 (blue) in the VPMNPL of 90-week-old KO mice (scale bar = $20 \mu \mathrm{m}$ ). D, Phospho-TDP-43 (pTDP-43)-immunostained image with Nissl counterstaining in the VPMNPL of 90-week-old KO mice (scale bar $=10 \mu \mathrm{m}$ ).

In the present study, we showed that aged PGRNdeficient mice presented with increased accumulation of p62 and ubiquitin in the VPM/VPL, where the main pathological changes have been observed in reported NCL model mice [15-18]. p62/ubiquitin inclusions have been observed when the autophagy-lysosomal system is disrupted [26]. Therefore, aged PGRN-deficient mice may have an impaired autophagy-lysosomal system. Since p62 inclusions were observed in neuronal and glial cells, PGRN may play a role in maintaining lysosomal function during aging in both neuronal and glial cells. Disruption of both the ubiquitin-proteasome system and the autophagy-lysosomal system is one of the characteristic features of FTLD with TDP-43 inclusions in the cytoplasm of neurons [27], and impaired production of PGRN is involved in this pathology [5-7]. Previous studies reported increased 


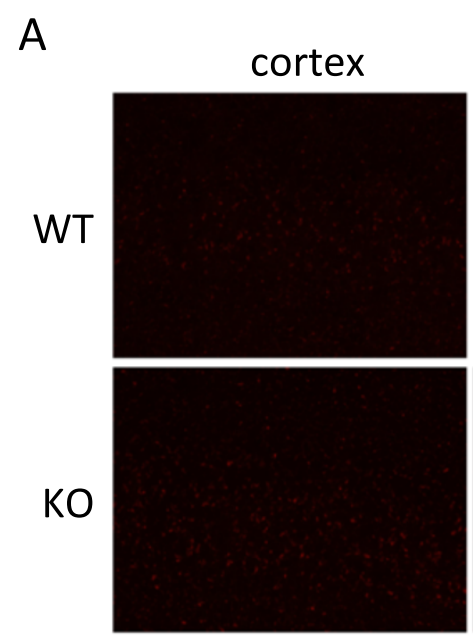

B

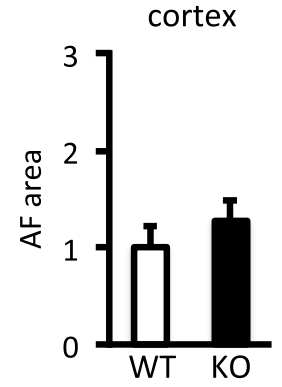

\section{hippocampus}
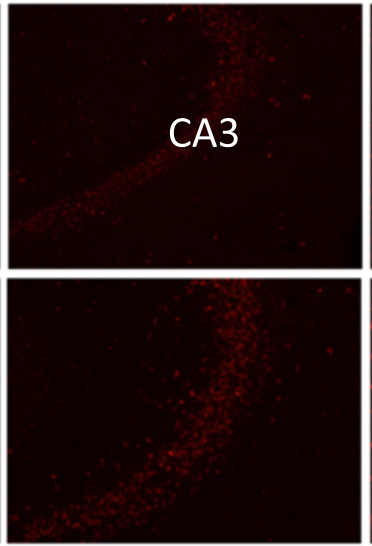

\section{VPM/VPL}
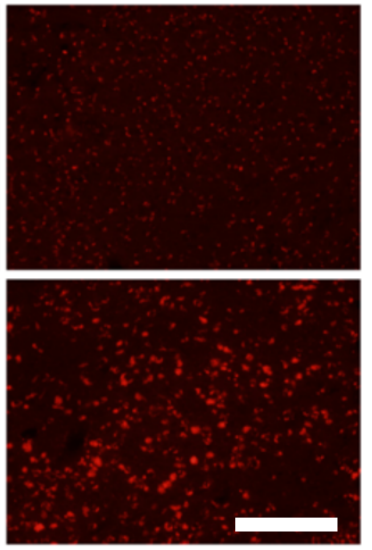

CA3

VPM/VPL
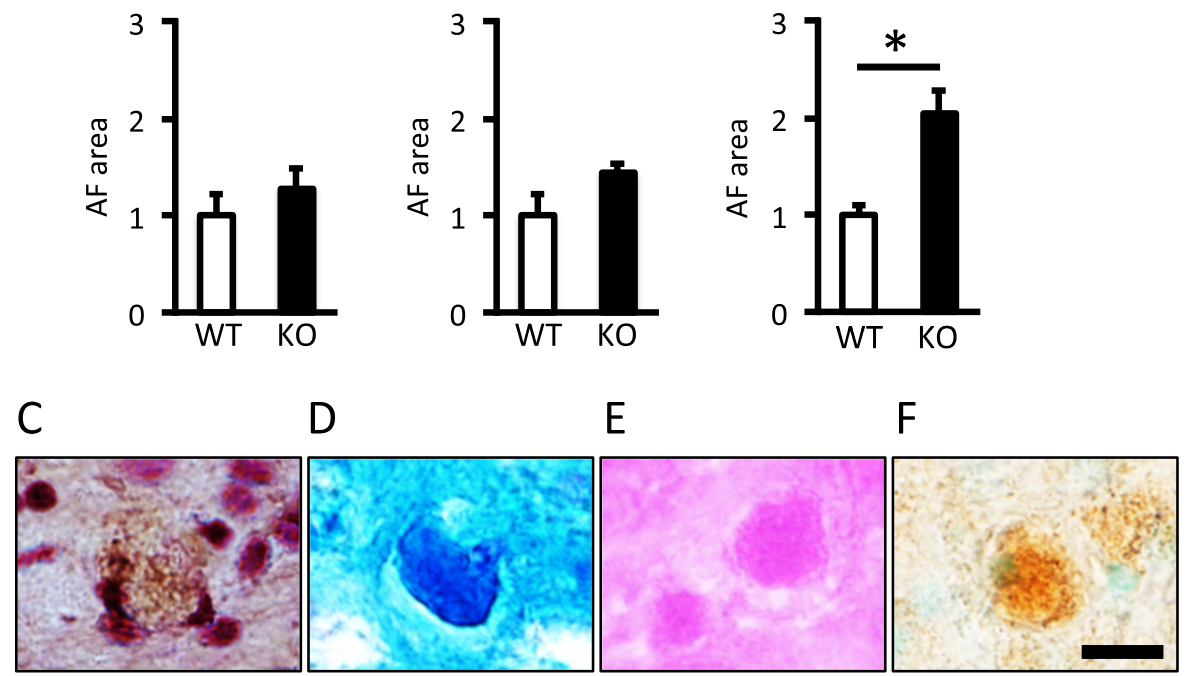

G
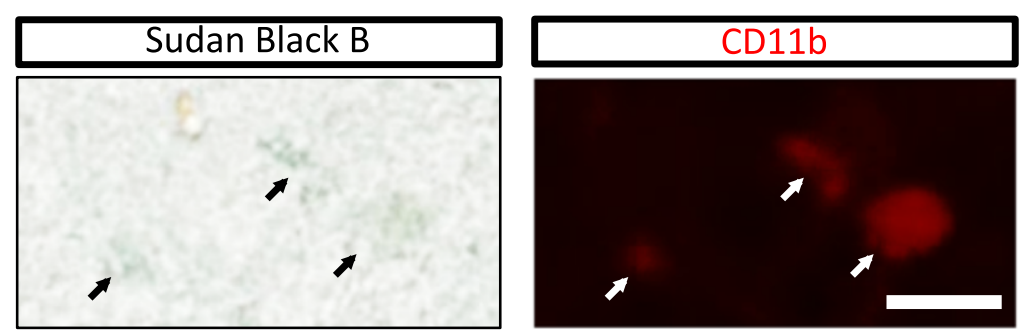

Figure 8 Increased lipofuscin accumulation in the VPM/VPL of aged PGRN-deficient mice. $\boldsymbol{A}$, Autofluorescence images of the cerebral cortex, hippocampus, and VPMNPL of 90-week-old wild-type (WT) and PGRN-deficient (KO) mice (scale bar $=200 \mu m)$. B, Autofluorescence (AF) area of the cerebral cortex, CA3 of the hippocampus, and VPMNPL of 90-week-old WT and KO mice. Values are the mean \pm SEM ( $n=5$ for each group, ${ }^{*} \mathrm{P}<0.05$, unpaired t-test). $\boldsymbol{C}, \boldsymbol{D}, \boldsymbol{E}, \boldsymbol{F}$, Hematoxylin-stained (C), Schmorl-stained (D), periodic acid-Schiff (PAS)-stained (E), and Oil-red-O-stained (F) images of the VPMNPL of 90-week-old KO mice (scale bar $=20 \mu \mathrm{m}$ ). $\mathbf{G}$, Images double-stained with Sudan black B and CD11b in the VPMNPL of 90-week-old KO mice (scale bar $=10 \mu \mathrm{m}$ ). Black and white arrows indicate Sudan black B-positive and CD11 b-positive cells, respectively.

TDP-43 phosphorylation in PGRN-deficient mice [11,13,28]. In the present study, TDP-43 colocalized with p62 in the cytoplasm of neurons as well as the phosphorylated TDP-43 aggregate in the VPM/VPL were only observed in aged
PGRN-deficient mice. Previous studies have shown that abnormal degradation by the autophagy-lysosomal pathway affects TDP-43 aggregation in the cytoplasm [29-32]. Taken together, these observations suggest that PGRN 


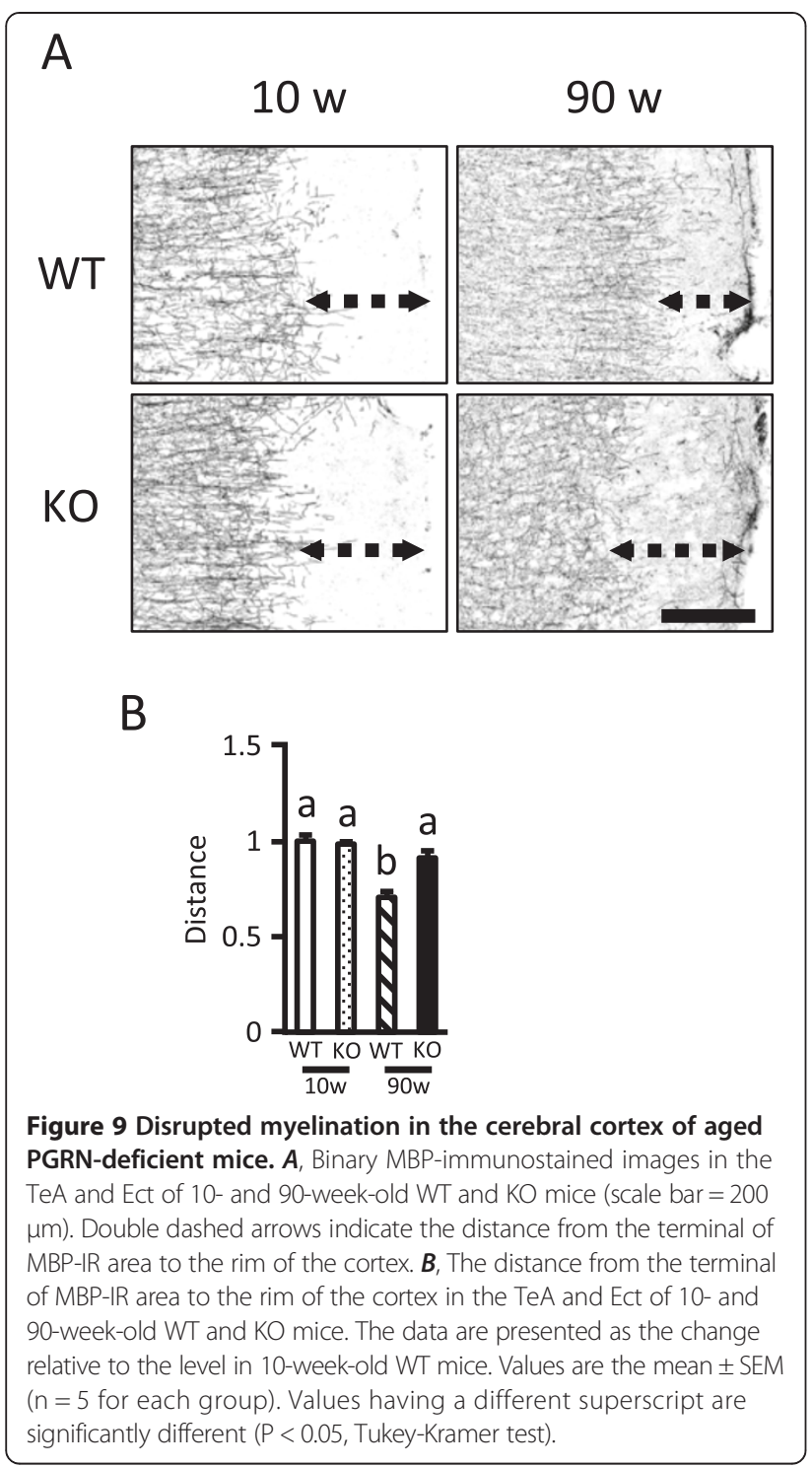

deficiency may be involved in generating TDP-43 aggregation in the cytoplasm associated with impaired cellular degradation by lysosomes.

We characterized the brain pathology related to NCL in aged PGRN-deficient mice in the present study. PGRN deficiency resulted in neuronal loss, increased gliosis, lipofuscin accumulation in the VPM/VPL, and defective myelination in the cerebral cortex. These pathological changes in aged PGRN-deficient mice are partially consistent with previous studies $[11,13,33]$, though the brain regions examined were different. Analyses using NCL model mice in the previous studies revealed that glial activation precedes neuronal loss in the VPM/VPL $[17,34]$. Therefore, the particular vulnerability of the VPM/VPL in PGRN-deficient mice might be attributed to cytotoxicity of glial cells. Since expression of genes for glial cell-derived neurotoxic factors was increased in the VPM/VPL of aged PGRN-deficient mice, PGRN deficiency is likely to make glial cells cytotoxic, thereby leading to enhanced neuroinflammation with aging, though further analysis on the vulnerability of the sensory thalamocortical pathways in PGRN-deficient mice might be needed. In addition, lipofuscin was accumulated in microglia of aged PGRN-deficient mice. Lipofuscin is thought to be an end product of cellular protein metabolism, especially protein catabolism [24]. Since macrophage in PGRN-deficient mice displayed enhanced rates of apoptotic-cell phagocytosis [35], microglia in PGRN-deficient mice might be more phagocytically active and thereby overloaded with waste products with a resultant increase in lipofuscin deposits. In the present study, defective myelination and enhanced astrogliosis occurred in the cerebral cortex of aged PGRN-deficient mice, but neuronal loss was not observed in the cerebral cortex. Similar observations were also made in 3-month-old mice lacking Cln1, a mutation in which causes classic infantile NCL [19]. Because astrocytes are implicated in myelination in the brain [36,37], PGRN deficiency might lead to impaired astrocyte function and consequently induce defective myelination, though further studies are necessary to elucidate myelination deficits due to PGRN deficiency. These observations indicate that our PGRN-deficient mice may be another useful experimental model for NCL.

Recently, it is reported that PGRN-deficient mice recapitulate pathobiomedical features of NCL and FTLD, e.g. increased lysosomal proteins and TDP-43 phosphorylation, and that FTLD patients due to PGRN deficiency have NCL-like pathology [13]. The present study also revealed that aged PGRN-deficient mice have characteristic features for both FTLD and NCL. Since lysosomal functions are involved in the pathogenesis of these diseases [13,38,39], increased lysosomal biogenesis and lysosomal dysfunction as observed in PGRN-deficient mice in the present study may be common processes in the pathogenic mechanism of these diseases. We have previously suggested that PGRN is localized in lysosomes and may be involved in the activation of mTORC1, which prevents translocation of TFEB to the nucleus by phosphorylation, thereby decreasing lysosomal biogenesis [9]. Further studies on the molecular mechanism underlying the actions of PGRN are needed to elucidate pathophysiological changes of the aged brain due to PGRN deficiency.

\section{Conclusions}

The present study demonstrated that aged PGRN-deficient mice show exacerbated neuronal loss, lipofuscin accumulation, gliosis, and increased lysosomal protein and 
gene expressions in the VPM/VPL of the thalamus, suggesting that aged PGRN-deficient mice develop NCLlike pathology. In addition, the presence of p62 aggregates in the VPM/VPL of aged PGRN-deficient mice suggests that TDP-43 aggregates observed in the cytoplasm of neurons in this brain region are associated with impaired cellular degradation by lysosomal dysfunction. To our knowledge, this is the first report showing NCL-like pathology and TDP-43 aggregates in the VPM/VPL of aged PGRN-deficient mice. Clarifying the mechanisms by which PGRN regulates lysosomal function may be crucial to elucidate pathoetiology of brain disorders resulting from PGRN deficiency.

\section{Additional file}

Additional file 1: Table S1. The primer list used in the present study.

\section{Abbreviations}

PGRN: Progranulin; FTLD: Frontotemporal lobar degeneration; NCL: Neuronal ceroid lipofuscinosis; WT: Wild-type; KO: PGRN-deficient; VPM/VPL: Ventral posteromedial nucleus/ventral posterolateral nucleus; TDP-43: TAR DNA binding protein 43; pTDP-43: Phospho-TAR DNA binding protein 43; TBI: Traumatic brain injury; S1BF: Primary somatosensory barrelfield cortex; RT: Room temperature; DW: Distilled water; IR: Immunoreactive; FD: Fractal dimension; PBST: PBS containing Triton X-100; TeA: Temporal association cortex; Ect: Ectorhinal cortex; mTORC1: Mammalian target of rapamycin complex 1.

\section{Competing interests}

The authors declare that they have no competing interests.

\section{Authors' contributions}

YT designed the present study, carried out the experiments and analyzed the data. YT also drafted the initial manuscript. JKC carried out lipofuscin analysis, microscopic observation and helped to draft the manuscript. TM and KY participated in the design of the study and helped to draft the manuscript. MN supervised the present study, and worked up the manuscript. All the authors read and approved the final manuscript.

\section{Authors' information}

YT is a JSPS postdoctoral research fellow, Department of Neuropathology and Cell Biology, Tokyo Metropolitan Institute of Medical Science. JKC is an assistant Professor, Department of Veterinary Pathology, The University of Tokyo. TM is an assistant Professor, Department of Veterinary Physiology, The University of Tokyo. KY is an associate Professor, Department of Veterinary Physiology, The University of Tokyo. MN is a Professor, Department of Veterinary Physiology, The University of Tokyo.

\section{Acknowledgements}

We are grateful to Sei Sato, Kazunori Sato, and Atsuhiro Tsutiya in the Graduate School of Life Sciences, Toyo University, Japan for technical help using the confocal microscope. This work was supported in part by JSPS KAKENHI Grant Number 23228004 to MN and 24-7548 to YT.

\section{Author details}

'Department of Veterinary Physiology, Graduate School of Agricultural and Life Sciences, The University of Tokyo, Bunkyo-ku, Tokyo 113-8657, Japan. ${ }^{2}$ Department of Neuropathology and Cell Biology, Tokyo Metropolitan Institute of Medical Science, 2-1-6 Kamikitazawa, Setagaya-ku, Tokyo 156-8506, Japan. ${ }^{3}$ Department of Veterinary Pathology, Graduate School of Agricultural and Life Sciences, The University of Tokyo, Bunkyo-ku, Tokyo 113-8657, Japan
Received: 16 May 2014 Accepted: 22 June 2014

Published: 15 July 2014

\section{References}

1. Cenik B, Sephton CF, Kutluk Cenik B, Herz J, Yu G: Progranulin: a proteolytically processed protein at the crossroads of inflammation and neurodegeneration. J Biol Chem 2012, 287:32298-32306.

2. Bateman A, Bennett HP: The granulin gene family: from cancer to dementia. Bioessays 2009, 31:1245-1254.

3. Thurner L, Preuss KD, Fadle N, Regitz E, Klemm P, Zaks M, Kemele M, Hasenfus A, Csernok E, Gross WL, Pasquali JL, Martin T, Bohle RM, Pfreundschuh M: Progranulin antibodies in autoimmune diseases. J Autoimmun 2013, 42:29-38.

4. Matsubara T, Mita A, Minami K, Hosooka T, Kitazawa S, Takahashi K, Tamori Y, Yokoi N, Watanabe M, Matsuo E, Nishimura O, Seino S: PGRN is a key adipokine mediating high fat diet-induced insulin resistanceand obesity through IL-6 in adipose tissue. Cell Metab 2012, 15:38-50.

5. Baker M, Mackenzie IR, Pickering-Brown SM, Gass J, Rademakers R, Lindholm C, Snowden J, Adamson J, Sadovnick AD, Rollinson S, Cannon A, Dwosh E, Neary D, Melquist S, Richardson A, Dickson D, Berger Z, Eriksen J, Robinson T, Zehr C, Dickey CA, Crook R, McGowan E, Mann D, Boeve B, Feldman H, Hutton M: Mutations in progranulin cause tau-negative frontotemporal dementia linked to chromosome 17. Nature 2006, 442:916-919.

6. Cruts M, Gijselinck I, van der Zee J, Engelborghs S, Wils H, Pirici D, Rademakers R, Vandenberghe R, Dermaut B, Martin JJ, van Duijn C, Peeters K, Sciot R, Santens P, De Pooter T, Mattheijssens M, Van den Broeck M, Cuijt I, Vennekens K, De Deyn PP, Kumar-Singh S, Van Broeckhoven C: Null mutations in progranulin cause ubiquitin-positive frontotemporal dementia linked to chromosome 17q21. Nature 2006, 442:920-924.

7. Arai T, Hasegawa M, Akiyama H, Ikeda K, Nonaka T, Mori H, Mann D, Tsuchiya K, Yoshida M, Hashizume Y, Oda T: TDP-43 is a component of ubiquitin-positive tau-negative inclusions in frontotemporal lobar degeneration and amyotrophic lateral sclerosis. Biochem Biophys Res Commun 2006, 351:602-611.

8. Tanaka Y, Matsuwaki T, Yamanouchi K, Nishihara M: Exacerbated inflammatory responses related to activated microglia after traumatic brain injury in progranulin-deficient mice. Neuroscience 2013, 231:49-60.

9. Tanaka Y, Matsuwaki T, Yamanouchi K, Nishihara M: Increased lysosomal biogenesis in activated microglia and exacerbated neuronal damage after traumatic brain injury in progranulin-deficient mice. Neuroscience 2013, 250:8-19.

10. Conde JR, Streit WJ: Microglia in the aging brain. J Neuropathol Exp Neurol 2006, 65:199-203.

11. Wils H, Kleinberger G, Pereson S, Janssens J, Capell A, Van Dam D, Cuijt I, Joris G, De Deyn PP, Haass C, Van Broeckhoven C, Kumar-Singh S: Cellular ageing, increased mortality and FTLD-TDP-associated neuropathology in progranulin knockout mice. J Pathol 2012, 228:67-76.

12. Smith KR, Damiano J, Franceschetti S, Carpenter S, Canafoglia L, Morbin M, Rossi G, Pareyson D, Mole SE, Staropoli JF, Sims KB, Lewis J, Lin WL, Dickson DW, Dahl HH, Bahlo M, Berkovic SF: Strikingly different clinicopathological phenotypes determined by progranulin-mutation dosage. Am J Hum Genet 2012, 90:1102-1107.

13. Götzl JK, Damme M, Fellerer K, Tahirovic S, Kleinberger G, Janssens J, Van Der Zee J, Lang CM, Kremmer E, Martin JJ, Engelborghs S, Kretzschmar HA, Arzberger T, Van Broeckhoven C, Haass C, Capell A: Common pathobiochemical hallmarks of progranulin-associated frontotemporal lobar degeneration and neuronal ceroid lipofuscinosis. Acta Neuropathol 2014. in press.

14. Jalanko A, Braulke T: Neuronal ceroid lipofuscinoses. Biochim Biophys Acta 2009, 1793:697-709.

15. Cooper JD: The neuronal ceroid lipofuscinoses: the same, but different? Biochem Soc Trans 2010, 38:1448-1452.

16. Kielar C, Maddox L, Bible E, Pontikis CC, Macauley SL, Griffey MA, Wong M, Sands MS, Cooper JD: Successive neuron loss in the thalamus and cortex in a mouse model of infantile neuronal ceroid lipofuscinosis. Neurobiol Dis 2007, 25:150-162.

17. Partanen S, Haapanen A, Kielar C, Pontikis C, Alexander N, Inkinen T, Saftig P, Gillingwater TH, Cooper JD, Tyynelä J: Synaptic changes in the thalamocortical system of cathepsin D-deficient mice: a model of human congenital neuronal ceroid-lipofuscinosis. J Neuropathol Exp Neurol 2008, 67:16-29. 
18. von Schantz C, Kielar C, Hansen SN, Pontikis CC, Alexander NA, Kopra O, Jalanko A, Cooper JD: Progressive thalamocortical neuron loss in Cln5 deficient mice: Distinct effects in Finnish variant late infantile NCL. Neurobiol Dis 2009, 34:308-319.

19. Blom T, Schmiedt ML, Wong AM, Kyttälä A, Soronen J, Jauhiainen M, Tyynelä J, Cooper JD, Jalanko A: Exacerbated neuronal ceroid lipofuscinosis phenotype in Cln1/5 double-knockout mice. Dis Model Mech 2013, 6:342-357.

20. Kuronen M, Hermansson M, Manninen O, Zech I, Talvitie M, Laitinen T, Gröhn O, Somerharju P, Eckhardt M, Cooper JD, Lehesjoki AE, Lahtinen U, Kopra O: Galactolipid deficiency in the early pathogenesis of neuronal ceroid lipofuscinosis model $\operatorname{Cln} 8^{\text {mnd }}$ : implications to delayed myelination and oligodendrocyte maturation. Neuropathol Appl Neurobiol 2012, 38:471-486

21. Kayasuga Y, Chiba S, Suzuki M, Kikusui T, Matsuwaki T, Yamanouchi K, Kotaki H, Horai R, Iwakura Y, Nishihara M: Alteration of behavioural phenotype in mice by targeted disruption of the progranulin gene. Behav Brain Res 2007, 185:110-118.

22. Oliveira VC, Carrara RC, Simoes DL, Saggioro FP, Carlotti CG Jr, Covas DT, Neder L: Sudan Black B treatment reduces autofluorescence and improves resolution of in situ hybridization specific fluorescent signals of brain sections. Histol Histopathol 2010, 25:1017-1024.

23. Sofroniew MV, Vinters HV: Astrocytes: biology and pathology. Acto Neuropathol 2010, 119:7-35.

24. Boellaard JW, Schlote W, Hofer W: Species-specific ultrastructure of neuronal lipofuscin in hippocampus and neocortex of subhuman mammals and humans. Ultrastruct Pathol 2004, 28:341-351.

25. Boggs JM: Myelin basic protein: a multifunctional protein. Cell Mol Life Sci 2006, 63:1945-1961.

26. Ferguson CJ, Lenk GM, Meisler MH: Defective autophagy in neurons and astrocytes from mice deficient in PI(3,5)P2. Hum Mol Genet 2009, 18:4868-4878.

27. Ju JS, Weihl CC: Inclusion body myopathy, Paget's disease of the bone and fronto-temporal dementia: a disorder of autophagy. Hum Mol Genet 2010, 19:R38-R45.

28. Yin F, Dumont M, Banerjee R, Ma Y, Li H, Lin MT, Beal MF, Nathan C, Thomas B, Ding A: Behavioral deficits and progressive neuropathology in progranulin-deficient mice: a mouse model of frontotemporal dementia. FASEB J 2010, 24:4639-4647.

29. Filimonenko M, Stuffers S, Raiborg C, Yamamoto A, Malerød L, Fisher EM, Isaacs A, Brech A, Stenmark H, Simonsen A: Functional multivesicular bodies are required for autophagic clearance of protein aggregates associated with neurodegenerative disease. J Cell Biol 2007, 179:485-500

30. Wang $X$, Fan H, Ying Z, Li B, Wang H, Wang G: Degradation of TDP-43 and its pathogenic form by autophagy and the ubiquitin-proteasome system. Neurosci Lett 2010, 469:112-116.

31. Caccamo A, Majumder S, Deng JJ, Bai Y, Thornton FB, Oddo S: Rapamycin rescues TDP-43 mislocalization and the associated low molecular mass neurofilament instability. J Biol Chem 2009, 284:27416-27424.

32. Gomes C, Escrevente C, Costa J: Mutant superoxide dismutase 1 overexpression in NSC-34 cells: effect of trehalose on aggregation, TDP-43 localization and levels of co-expressed glycoproteins. Neurosci Lett 2010, 475:145-149.

33. Ahmed Z, Sheng H, Xu YF, Lin WL, Innes AE, Gass J, Yu X, Wuertzer CA Hou H, Chiba S, Yamanouchi K, Leissring M, Petrucelli L, Nishihara M, Hutton ML, McGowan E, Dickson DW, Lewis J: Accelerated lipofuscinosis and ubiquitination in granulin knockout mice suggest a role for progranulin in successful aging. Am J Pathol 2010, 177:311-324.

34. Kuronen $M$, Lehesjoki AE, Jalanko A, Cooper JD, Kopra O: Selective spatiotemporal patterns of glial activation and neuron loss in the sensory thalamocortical pathways of neuronal ceroid lipofuscinosis 8 mice. Neurobiol Dis 2012, 47:444-457.

35. Kao AW, Eisenhut RJ, Martens LH, Nakamura A, Huang A, Bagley JA, Zhou P, de Luis A, Neukomm LJ, Cabello J, Farese RV Jr, Kenyon C: A neurodegenerative disease mutation that accelerates the clearance of apoptotic cells. Proc Natl Acad Sci U S A 2011, 108:4441-4446.

36. Schulz K, Kroner A, David S: Iron efflux from astrocytes plays a role in remyelination. J Neurosci 2012, 32:4841-4847.

37. Barateiro A, Domingues HS, Fernandes A, Relvas JB, Brites D: Rat cerebellar slice cultures exposed to bilirubin evidence reactive gliosis, excitotoxicity and impaired myelinogenesis that is prevented by AMPA and TNF- $a$ inhibitors. Mol Neurobiol 2014, 49:424-439.

38. Appelqvist $H$, Wäster $P$, Kågedal $K$, Ollinger $K$ : The lysosome: from waste bag to potential therapeutic target. J Mol Cell Biol 2013, 5:214-226.

39. Brady OA, Zheng Y, Murphy K, Huang M, Hu F: The frontotemporal lobar degeneration risk factor, TMEM106B, regulates lysosomal morphology and function. Hum Mol Genet 2013, 22:685-695.

doi:10.1186/s40478-014-0078-x

Cite this article as: Tanaka et al:: Possible involvement of lysosomal dysfunction in pathological changes of the brain in aged progranulin-deficient mice. Acta Neuropathologica Communications $20142: 78$

\section{Submit your next manuscript to BioMed Central and take full advantage of:}

- Convenient online submission

- Thorough peer review

- No space constraints or color figure charges

- Immediate publication on acceptance

- Inclusion in PubMed, CAS, Scopus and Google Scholar

- Research which is freely available for redistribution 